\title{
Baryogenesis in the standard model and its supersymmetric extension
}

\author{
Chee Sheng Fong $\odot^{*}$ \\ Centro de Ciências Naturais e Humanas Universidade Federal do ABC, \\ 09.210-170 Santo André, SP, Brazil
}

(Received 15 December 2020; accepted 10 March 2021; published 26 March 2021)

\begin{abstract}
In this work, we classify all the effective $U(1)$ symmetries and their associated Noether charges in the standard model (SM) and its minimal supersymmetric extension (MSSM) from the highest scale after inflation down to the weak scale. We then demonstrate that the discovery of the violation of baryon minus lepton number $(B-L)$, which pinpoints to its violation in the primordial Universe at any cosmic temperature above $30 \mathrm{TeV}$, will open up a new window of baryogenesis in these effective charges above this scale. While the fast SM baryon number violation in the early Universe could be the first piece to solve the puzzle of cosmic baryon asymmetry, $(B-L)$ violation could be the second important piece. In the background of an expanding Universe, there is ample opportunity for out-of-equilibrium processes to generate an asymmetry in the numerous effective charges in the SM or the MSSM, making a baryonasymmetric Universe almost unavoidable. Finally, we provide examples in the SM and the MSSM where baryogenesis can proceed through out-of-equilibrium dynamics without explicitly breaking baryon or lepton number.
\end{abstract}

DOI: 10.1103/PhysRevD.103.L051705

\section{INTRODUCTION}

Symmetry has been a guiding principle in physics. In the standard model $(\mathrm{SM})$, the gauge symmetry $S U(3)_{c} \times$ $S U(2)_{L} \times U(1)_{Y}$ fixes all the possible interactions among the fields. However, once one consider the same theory in an expanding Universe, new effective symmetries can arise if the rates of certain interactions are slower than the Hubble rate-i.e., out of thermal equilibrium. For instance, while baryon-number-violating interactions due to $S U(2)_{L}$ instanton-induced operators are suppressed to an unobservable rate today [1], they were in thermal equilibrium for cosmic temperatures above the weak scale [2]. This baryonnumber violation in the primordial Universe, among others, could be a key ingredient to understand the observed cosmic baryon asymmetry represented by the baryonnumber density asymmetry over the cosmic entropic density $Y_{\Delta B} \sim 9 \times 10^{-11}[3]$.

Symmetry in the context of baryogenesis is a doubleedged sword. On the one hand, if a symmetry remains exact, no asymmetry can be generated in the associated Noether charge. On the other hand, it can protect an asymmetry generated from being erased. This was first

\footnotetext{
*sheng.fong@ufabc.edu.br
}

Published by the American Physical Society under the terms of the Creative Commons Attribution 4.0 International license. Further distribution of this work must maintain attribution to the author(s) and the published article's title, journal citation, and DOI. Funded by SCOAP ${ }^{3}$. elucidated by Weinberg [4]: that as long as there is a linear combination of baryon and lepton number $B+a L$ that is conserved, a baryon asymmetry is preserved even if baryon-number-violating interactions are in equilibrium. The use of other types of symmetry as a protective mechanism was further explored in the SM [5-7], in its extension [8,9], and in its supersymmetric extension [10], and it was generalized in Ref. [11].

In this work, we will categorize all the effective symmetries and their associated Noether charges in the SM and the minimal supersymmetric standard model (MSSM), from the highest temperature after reheating, $T \sim 10^{16} \mathrm{GeV}$, down to the weak scale. Making use of $(B-L)$-violating interactions which are in equilibrium, we show that asymmetries can be generated in any of the effective charges through out-of-equilibrium processes which can, but do not have to violate baryon nor lepton number.

\section{GENERALITY}

Here we give a brief review of the formalism discussed in Ref. [11], which will be used in this work and also to fix the notations. For any particle species $i$ that distinguishes from its antiparticle $\bar{i}$-i.e., a "complex particle"-its number density asymmetry can be defined as $n_{\Delta i} \equiv n_{i}-n_{\bar{i}}$, where $n_{i}$ is the number density of particle $i$. This is equivalent to its charge density if we assign the particle (antiparticle) a charge $1(-1)$ under a global $U(1)_{i}$. Considering $r$ species of complex particles $i$, we can define $r$ number of such 
global $U(1)_{i}$ 's preserved by their kinetic terms (which include possible gauge interactions).

Next, let us put the system in an expanding Universe. Assuming that fast gauge interactions are able to thermalize the particles, a common temperature $T$ can be defined at each moment. To scale out the effect due to cosmic expansion, we define the number asymmetry of the particle $i$ as

$$
Y_{\Delta i}=\frac{n_{\Delta i}}{s},
$$

where $s=\frac{2 \pi}{45} g_{\star} T^{3}$ is the cosmic entropic density, with $g_{\star}$ being the total relativistic degrees of freedom. From $r$ number of $U(1)_{i}$ 's, we can form any other $r$ linear combinations $U(1)_{x}$. A convenient basis is such that as the expanding Universe is cooling down, $U(1)_{x}$ is subsequently broken by interactions which get into thermal equilibrium.

The source of $U(1)_{x}$ can be due to additional interactions like the Yukawa interactions or those from instantoninduced effective operators due to the Bell-Jackiw anomaly [1]. To determine if a $U(1)_{x}$ is preserved by the latter type of interactions, we can look at the anomaly coefficient of the triangle diagram of the type $U(1)_{x}-S U(N)-S U(N)$, defined as

$$
A_{x N N} \equiv \sum_{i} c\left(R_{i}\right) g_{i} q_{i}^{x}
$$

where the sum is over particle $i$ with degeneracy $g_{i}$, charge $q_{i}^{x}$ under $U(1)_{x}$, and representation $R_{i}$ under the $S U(N \geq 2)$ gauge group with $c\left(R_{i}\right)=\frac{1}{2}$ in the fundamental representation and $c_{2}\left(R_{i}\right)=N$ in the adjoint representation. In general, each fermion $i$ with representation $R_{i}$ under $S U(N)$ will contribute proportionally to $c\left(R_{i}\right)$ to the $S U(N)$ instanton-induced effective operator ${ }^{1}$

$$
\mathcal{O}_{S U(N)} \sim \prod_{i} \Psi_{i}^{2 g_{i} c\left(R_{i}\right)}
$$

where the product is over all left-handed chiral fields $\Psi_{i}$ with nontrivial representation $R_{i}$ under $S U(N)$. Notice that if $A_{x N N}=0, U(1)_{x}$ is conserved by the operator.

If a system possesses a $U(1)_{x}$, its charge can be written as

$$
Y_{\Delta x}=\sum_{i} q_{i}^{x} Y_{\Delta i}
$$

where $i$ sums over all particle species with charge $q_{i}^{x}$ under $U(1)_{x}$. Assuming equilibrium phase-space distribution for particle $i$ characterized by a common temperature $T$, its

\footnotetext{
${ }^{1}$ Due to the convention $c\left(R_{i}\right)=\frac{1}{2}$ for fundamental representation, the factor of 2 is included such that the field enters in an integer number.
}

energy $E_{i}$, and chemical potential $\mu_{i} \ll T$, the formula above can be inverted as [11]

$$
Y_{\Delta i}=\sum_{x} g_{i} \zeta_{i} \sum_{y} q_{i}^{y}\left(J^{-1}\right)_{y x} Y_{\Delta x}
$$

where $\zeta_{i}=1$ (2), where $i$ is a massless fermion (boson), ${ }^{2}$ and $J$ is a symmetric matrix in charge space defined as

$$
J_{x y} \equiv \sum_{i} g_{i} \zeta_{i} q_{i}^{x} q_{i}^{y}
$$

From Eq. (5), we can construct the baryonic charge as

$$
Y_{\Delta B}=\sum_{i} q_{i}^{B} Y_{\Delta i}=\sum_{x} \sum_{y} J_{B y}\left(J^{-1}\right)_{y x} Y_{\Delta x} .
$$

It is apparent that the cosmic baryon asymmetry is proportional to the $U(1)_{x}$ charges of the system. Next, our goal is to characterize all the $U(1)_{x}$ 's in the SM and MSSM.

\section{THE STANDARD MODEL}

Before the electroweak (EW) symmetry breaking, the SM kinetic terms respect a total of $16 U(1)_{\Psi_{j}}$ 's, each corresponding to the individual field rotation of the 15 fermionic fields and a scalar Higgs $S U(2)_{L}$ doublet $H: \Psi_{j} \rightarrow e^{i q_{\Psi_{j}} \phi} \Psi_{j}$, where $\Psi=\left\{Q_{\alpha}, U_{\alpha}, D_{\alpha}, \ell_{\alpha}, E_{\alpha}, H\right\}$ with family index $\alpha=1,2,3$. Here $Q_{\alpha}$ and $\ell_{\alpha}$ are the quark and lepton $S U(2)_{L}$ doublets, while $U_{\alpha}, D_{\alpha}$, and $E_{\alpha}$ are the up-type quark, down-type quark, and lepton $S U(2)_{L}$ singlets, respectively. ${ }^{3}$ Since all the parameters of the SM have been measured, we can choose the following convenient basis according to the order in which the $U(1)_{x}$ symmetries are subsequently broken at $T_{x}$ as we go down in the cosmic temperature:

$$
\begin{gathered}
x=\left\{t, u, B, u-b, \tau, u-c, \mu, B_{3}-B_{2}, u-s,\right. \\
\left.B_{3}+B_{2}-2 B_{1}, u-d, e, B / 3-L_{\alpha}, Y\right\} .
\end{gathered}
$$

$U(1)_{B_{\alpha}}$ corresponds to baryon flavor symmetries, with $q_{Q_{\alpha}, U_{\alpha}, D_{\alpha}}^{B_{\alpha}}=1 / 3$, and $U(1)_{B} \equiv U(1)_{B_{1}+B_{2}+B_{3}}$ is the total baryon number. $U(1)_{B / 3-L_{\alpha}}$ is the linear combination of the $B / 3$ and lepton flavor symmetries $U(1)_{L_{\alpha}}$, with $q_{\ell_{\alpha}, E_{\alpha}}^{L_{\alpha}}=1$. The rest of the global charges are normalized to 1 .

$U(1)_{Y}$ is the hypercharge gauge symmetry, which is only broken at $T_{\mathrm{EW}} \sim 160 \mathrm{GeV}$ [12], while the rest are effective (global) symmetries which are broken at $T_{t} \sim 10^{15} \mathrm{GeV}$, $T_{u} \sim 2 \times 10^{13} \mathrm{GeV}, T_{B} \sim 2 \times 10^{12} \mathrm{GeV}, T_{\tau} \sim 4 \times 10^{11} \mathrm{GeV}$, $T_{u-b} \sim 3 \times 10^{11} \mathrm{GeV}, T_{u-c} \sim 2 \times 10^{10} \mathrm{GeV}, T_{\mu} \sim 10^{9} \mathrm{GeV}$,

\footnotetext{
${ }^{2}$ For a particle with mass $m_{i}, \zeta_{i}=\frac{6}{\pi^{2}} \int_{m_{i} / T}^{\infty} d x x \sqrt{x^{2}-m_{i}^{2} / T^{2}} \frac{e^{x}}{\left(e^{x} \pm \xi_{i}\right)^{2}}$, where $\xi_{i}=1(-1)$ where $i$ is a fermion (boson).

${ }^{3}$ We will also denote $\left\{U_{1}, U_{2}, U_{3}\right\}=\{u, c, t\},\left\{D_{1}, D_{2}, D_{3}\right\}=$ $\{d, s, b\}$, and $\left\{E_{1}, E_{2}, E_{3}\right\}=\{e, \mu, \tau\}$.
} 
$T_{B_{3}-B_{2}} \sim 9 \times 10^{8} \mathrm{GeV}, T_{u-s} \sim 3 \times 10^{8} \mathrm{GeV}, T_{B_{3}+B_{2}-2 B_{1}} \sim$ $10^{7} \mathrm{GeV}, T_{u-d} \sim 2 \times 10^{6} \mathrm{GeV}$, and $T_{e} \sim 3 \times 10^{4} \mathrm{GeV}$. The estimations of $T_{x}$ are explained in the Supplemental Material [13]. Once broken at $T_{x}$, the symmetries are not restored again, with the exception of $U(1)_{B}$. From Eq. (3), we have the $S U(2)_{L}$ instanton-induced operator $\mathcal{O}_{S U(2)_{L}} \sim \prod_{\alpha}(Q \ell \ell \ell)_{\alpha}$, which violates $U(1)_{B}$, and the associated processes are in thermal equilibrium [2] from $T_{B} \sim 2 \times 10^{12} \mathrm{GeV}$ [14] down to $T_{B-} \sim 130 \mathrm{GeV}$ [12]. This is the source of baryon-number violation for EW baryogenesis [15], though in the SM, two other Sakharov's conditions for baryogenesis [16]—sufficient $\mathrm{C}$ and $C P$ violation $[17,18]$, and sufficiently out-of-equilibrium processes [19]—are not fulfilled.

Among the global symmetries, only $U(1)_{B / 3-L_{\alpha}}$ (or any linear combinations) are exact: they are conserved by all the Yukawa interactions and free from $S U(2)_{L}$ and $S U(3)_{c}$ mixed anomalies. At temperatures below $T_{e} \sim 30 \mathrm{TeV}$, only $U(1)_{Y}$ and $U(1)_{B / 3-L_{\alpha}}$ remain conserved, and from Eq. (7), we have

$$
Y_{\Delta B}=c_{B(B-L)} Y_{\Delta(B-L)}+c_{B Y} Y_{\Delta Y}
$$

where $Y_{\Delta(B-L)}=\sum_{\alpha} Y_{\Delta\left(B / 3-L_{\alpha}\right)}$ and $c_{B x} \equiv \sum_{y} J_{B y}\left(J^{-1}\right)_{y x}$, with the $J$ matrix defined in Eq. (6). Assuming the EW symmetry is broken at $T_{\mathrm{EW}} \sim 160 \mathrm{GeV}$ above $T_{B-}$ [12], we have $c_{B(B-L)}=\frac{30}{97}$ and $c_{B Y}=-\frac{7}{97}$ assuming that top quarks are nonrelativistic (cf. Ref. [20]). Equation (9) holds only down to temperature $T_{B-}$, below which the baryon number is frozen. If the Universe is always hypercharge neutral, $Y_{\Delta Y}=0$, this implies that $U(1)_{B-L}$ has to be broken above $T_{B-}$ to generate a nonzero baryon asymmetry, as utilized in leptogenesis [21], its variants [22,23], and $\mathrm{SO}(10)$ baryogenesis [24]. ${ }^{4}$ With $Y_{\Delta Y}=0$ and in the absence of new charges, if $(B-L)$-violating interactions remain in thermal equilibrium from $T_{e}$ down until $T_{B-}$, baryogenesis will fail. $^{5}$

It is usually required that $(B-L)$-violating interactions from new physics be out of equilibrium for a viable baryogenesis scenario (see, for example, Refs. [20,33]). In this work, we will point out an orthogonal scenario. Rather, we argue that any in-equilibrium $(B-L)$-violating interactions in fact facilitate baryogenesis and allow a new avenue of baryogenesis through out-of-equilibrium generation of asymmetry in the effective charges identified in Eq. (8).

\footnotetext{
${ }^{4}$ Extending the SM by new fields which carry nonzero $(B-L)$ charges, baryogenesis can proceed with unbroken $U(1)_{B-L}$ [9,25-28]. Some of this type of model can also accommodate the situation where the compensating $(B-L)$ charge remains in the hidden sector and can serve as dark matter $[27,28]$.

${ }^{5}$ See Refs. [29-32] for studies to bound the scale of baryogenesis due to fast $(B-L)$-violating interactions.
}

In general, fast $(B-L)$-violating interactions are more than welcome, since they will act as the source of nonzero $(B-L)$ charge in Eq. (9) [34]. The indication that $U(1)_{B-L}$ is broken from new physics is ubiquitous. If the SM is treated as an effective field theory at low energy, at mass dimension 5, we have the Weinberg operator $\ell_{\alpha} H \ell_{\beta} H$, which breaks $(B-L)$ by 2 units and gives rise to Majorana neutrino mass at low energy $[4,35]$. It has been verified that all dimension-6 [4,35] and dimension-8 operators [36] conserve $(B-L)$, while for the 18 dimension-7 [37,38] and 560 dimension-9 operators [39,40], $(B-L)$ is violated by 2 units. If $\Delta B=-\Delta L=1$, they lead to nucleon decay channels on top of those from the operators that conserve $B-L$ [4]. If $\Delta L=2$, these operators contribute to Majorana neutrino mass and neutrinoless double beta decay processes $[4,35,41,42]$, while if $\Delta B=2$, they can lead to neutron-antineutron oscillation (see Ref. [43] for a review article). Finally, a gauge $U(1)_{B-L}$ naturally arises from gauge symmetry $S O(10)$ in grand unified theory and is broken spontaneously to the SM gauge group.

If any of the $(B-L)$-violating processes discussed above are in thermal equilibrium in a certain temperature regime, we can construct $(B-L)$ charge asymmetry as

$$
Y_{\Delta(B-L)}=\sum_{x} c_{(B-L) x} Y_{\Delta x}
$$

where $c_{(B-L) x}=\sum_{y} J_{(B-L) y}\left(J^{-1}\right)_{y x}$, with the $J$ matrix defined in Eq. (6). At any range of temperature regime when $(B-L)$-violating interactions are in thermal equilibrium, a nonzero $Y_{\Delta(B-L)}$ is induced as long as any $x$ with nonzero coefficient $c_{(B-L) x} \neq 0$ has a nonvanishing charge $Y_{\Delta x} \neq 0$, which can be generated through out-of-equilibrium dynamics at the same range of temperature or much before (at higher temperature). As long as $(B-L)$-violating processes get out of equilibrium shortly after, the final baryon asymmetry will be given by Eq. (9).

To recapitulate, this new class of baryogenesis can be realized by extending the SM with the following:

(1) Some in-equilibrium $(B-L)$-violating interactions to enforce Eq. (10).

(2) Some out-of-equilibrium processes that violate the effective symmetries in Eq. (8) and also provide sources of $\mathrm{C}$ and $C P$ violation. In fact, all the global charges (besides $B-L$ ) are violated explicitly in the $\mathrm{SM}$, but new physics is required to have sufficient $C P$ violation and out-of-equilibrium condition.

As a concrete example, let us consider the temperature regime $10^{12} \mathrm{GeV} \lesssim T \lesssim 10^{14} \mathrm{GeV}$, where the $(B-L)$ violating interactions mediated by the Weinberg operator $\frac{1}{\Lambda} \ell_{\alpha} H \ell_{\beta} H$ are in thermal equilibrium. The estimation goes as follows: with the neutrino mass $m_{\nu}=v^{2} / \Lambda$ where $v \equiv\langle H\rangle=174 \mathrm{GeV}$, this implies $\Lambda \sim 3 \times 10^{14}\left(\frac{0.1 \mathrm{eV}}{m_{\nu}}\right) \mathrm{GeV}$. Comparing the $(B-L)$-violating rate $\Gamma_{B-L} \sim m_{\nu}^{2} T^{3} / v^{4}$ 
for $T \lesssim \lambda^{2} \Lambda$ (where $\lambda$ is some dimensionless coupling) to the Hubble rate $H=1.66 \sqrt{g_{\star}} T^{2} / M_{\mathrm{Pl}}$ (where $g_{\star}=106.75$ for the SM and $\left.M_{\mathrm{Pl}}=1.22 \times 10^{19} \mathrm{GeV}\right)$, we obtain $T_{B-L} \sim$ $10^{11}\left(\frac{0.1 \mathrm{eV}}{m_{\nu}}\right)^{2} \mathrm{GeV}$, above which the $(B-L)$-violating interactions are in equilibrium as long as $\lambda \gtrsim 0.02\left(\frac{0.1 \mathrm{eV}}{m_{\nu}}\right)$.

Next, let us introduce some processes which violate the effective symmetries of the SM. In general, grand unified theories contain various such possibilities. For instance, the 126 Higgs in $S O(10)$ contains diquarks, dileptons, and leptoquarks, and their couplings to the SM fields violate several of the effective charges in Eq. (8). As an example, let us introduce a heavy $S U(2)_{L}$ singlet colored diquark $\psi$ with $B=2 / 3, \quad Y=-2 / 3$, and the decay channels $\psi \rightarrow d d, s s, b b$, which violate $U(1)_{u-d}, U(1)_{u-s}$, and $U(1)_{u-b}$, respectively, by -2 units while respecting all other $U(1)_{x}$ symmetries (including $B$ and $L$ ). ${ }^{6}$ [A nonzero charge will be induced in $U(1)_{\psi}$ during the genesis, but this will go to zero once all $\psi$ particles have decayed away.] Assuming $C P T$ invariance, the $C P$ violations from the decays are related by

$$
\epsilon_{\psi \rightarrow d d}+\epsilon_{\psi \rightarrow s s}+\epsilon_{\psi \rightarrow b b}=0,
$$

where we have defined the $C P$ parameter as $\epsilon_{\psi \rightarrow j} \equiv$ $\frac{\Gamma(\psi \rightarrow j)-\Gamma(\bar{\psi} \rightarrow \bar{j})}{\Gamma_{\psi}}$, with $\Gamma_{\psi}$ being the total decay width of $\psi$ and $\Gamma(\psi \rightarrow j)$ and $\Gamma(\bar{\psi} \rightarrow \bar{j})$ being the partial decay widths. The charge $Y_{\Delta x}$ generated from the decays of $\psi$ can be parametrized by

$$
Y_{\Delta x}=-2 \epsilon_{\psi(x)} \eta_{x} Y_{\psi}^{\mathrm{eq}},
$$

where $\psi(x)$ refers to the decay process which violates $x$ charge, $\eta_{x} \leq 1$ is the efficiency for $x$-charge production through out-of-equilibrium dynamics, and $Y_{\psi}^{\mathrm{eq}}=n_{\psi}^{\mathrm{eq}} / s$, with $n_{\psi}^{\text {eq }}$ being the relativistic equilibrium number density of $\psi$. If $\psi$ particles start from a thermal abundance and decay far from equilibrium when $T \ll m_{\psi}$, we have $\eta_{u-d}=\eta_{u-s}=\eta_{u-b}=1$. Making use of Eqs. (10) and (11) and with all conserved charges remaining zero, after all $\psi$ particles have decayed above $T \sim 10^{12} \mathrm{GeV}$, we end up with

$$
Y_{\Delta(B-L)}=\left\{\begin{array}{ll}
\frac{1}{3} \epsilon_{\psi \rightarrow b b} Y_{\psi}^{\mathrm{eq}} & T_{u}<T<T_{t} \\
\frac{9}{22} \epsilon_{\psi \rightarrow b b} Y_{\psi}^{\mathrm{eq}} & T_{B}<T<T_{u}
\end{array} .\right.
$$

Below $T_{B-L},(B-L)$ is conserved, and the final baryon asymmetry is given by Eq. (9) with $Y_{\Delta Y}=0$. In order to obtain $Y_{\Delta B} \sim 10^{-10}$ in accordance with observation [3], since $Y_{\psi}^{\mathrm{eq}} \sim 10^{-3}$, one would need a reasonable $C P$ violation of $\epsilon_{\psi \rightarrow b b} \sim 10^{-6}$.

\footnotetext{
${ }^{6}$ For simplicity, we assume the decay channels are flavor diagonal.
}

\section{THE MINIMAL SUPERSYMMETRIC SM}

In the MSSM, all the SM fermionic fields are promoted to superfields. For anomaly cancellation, two Higgs superfields $H_{u}$ and $H_{d}$ are introduced, and we can choose the additional $U(1)_{P Q}$ conserved by all the superpotential terms $^{7}$

$$
\begin{aligned}
W= & \mu H_{u} H_{d}+\left(y_{u}\right)_{\alpha \beta} Q_{\alpha} H_{u} U_{\beta}^{c}+\left(y_{d}\right)_{\alpha \beta} Q_{\alpha} H_{d} D_{\beta}^{c} \\
& +\left(y_{e}\right)_{\alpha \beta} \ell_{\alpha} H_{u} E_{\beta}^{c}
\end{aligned}
$$

except $\mu H_{u} H_{d}$ with the following charge assignments:

$$
\begin{aligned}
& q_{H_{d}}^{P Q}=q_{\ell_{\alpha}}^{P Q}=-\frac{q_{E_{\alpha}^{c}}^{P Q}}{2}, \quad q_{H_{u}}^{P Q}=-\frac{q_{E_{\alpha}^{c}}^{P Q}}{2}+3 q_{D_{\alpha}^{c}}^{P Q}, \\
& q_{Q_{\alpha}}^{P Q}=\frac{q_{E_{\alpha}^{c}}^{P Q}}{2}-q_{D_{\alpha}^{c}}^{P Q}, \quad q_{U_{\alpha}^{c}}^{P Q}=-2 q_{D_{\alpha}^{c}}^{P Q} .
\end{aligned}
$$

The mixed anomaly coefficients of $U(1)_{P Q}$ with $S U(3)_{c}$ and $S U(2)_{L}$ are given by $A_{P Q 33}=\frac{3}{2}\left(-3 q_{D_{\alpha}^{c}}^{P Q}+q_{E_{\alpha}^{c}}^{P Q}\right)$ and $A_{P Q 22}=-3 q_{D_{\alpha}^{c}}^{P Q}+q_{E_{\alpha}^{c}}^{P Q}$, respectively. The anomaly-free choice $-3 q_{D_{\alpha}^{c}}^{P Q}+q_{E_{\alpha}^{c}}^{P Q}=0$ is the hypercharge, and hence we will consider only the solutions with $-3 q_{D_{\alpha}^{c}}^{P Q}+q_{E_{\alpha}^{c}}^{P Q} \neq 0$. Since both $B$ and $L$ have the same anomaly coefficient $A_{B 22}=A_{L 22}=\frac{3}{2}$, an $S U(2)_{L}$ mixed anomaly-free charge can be formed [11]:

$$
P=-\frac{3}{2} \frac{P Q}{-3 q_{D^{c}}^{P Q}+q_{E^{c}}^{P Q}}+\frac{1}{c_{B L}}\left(c_{B} B+c_{L} L\right),
$$

where $c_{B L}=c_{B}+c_{L}$ with $c_{B}$ and $c_{L}$ being any numbers. As for the $S U(3)_{c}$ mixed anomaly, we can cancel it with any of the chiral symmetries of the quark fields $U(1)_{Q_{\alpha}}$, $U(1)_{U_{\alpha}^{c}}$, and $U(1)_{D_{\alpha}^{c}}$ with the respective anomaly coefficients $A_{Q_{\alpha} 33}=1$ and $A_{U_{\alpha}^{c} 33}=A_{D_{\alpha}^{c} 33}=\frac{1}{2}$ (all the chiral charges are fixed to be 1). For instance, a completely anomaly-free combination will be

$$
\bar{P}=P+\frac{9}{2} u^{c} .
$$

Since $\bar{P}$ is violated explicitly only by the $\mu H_{u} H_{d}$ term, by comparing the interaction rate $\Gamma_{\bar{P}} \sim \mu^{2} / T$ to the Hubble rate $H=1.66 \sqrt{g_{\star}} T^{2} / M_{\mathrm{Pl}}\left(g_{\star}=228.75\right.$ for the MSSM $)$, we obtain [10]

$$
T_{\bar{P}} \sim 2 \times 10^{7}\left(\frac{\mu}{100 \mathrm{GeV}}\right)^{2 / 3} .
$$

\footnotetext{
${ }^{7}$ Here, we denote $\Psi=\left\{Q_{\alpha}, U_{\alpha}^{c}, D_{\alpha}^{c}, \ell_{\alpha}, E_{\alpha}^{c}, H_{u}, H_{d}\right\}$ as the left-handed chiral superfields.
} 
Above this temperature, $U(1)_{\bar{P}}$ is preserved by all the MSSM interactions.

In a supersymmetric theory, gauginos can carry nonvanishing chemical potentials, and scalar and fermionic components of a chiral superfield do not necessarily carry the same chemical potentials. This is captured by the $R$ symmetry, in which the superspace coordinate transforms as $\theta \rightarrow e^{i \phi} \theta$, where we fix its $R$ charge to be 1 . Requiring the superpotential in Eq. (14) to have an $R$ charge equal to 2 , we have

$$
\begin{aligned}
& q_{H_{d}}^{R}=q_{\ell_{\alpha}}^{R}=2-\frac{3 q_{D_{\alpha}^{c}}^{R}}{2}, \quad q_{H_{u}}^{R}=\frac{3 q_{D_{\alpha}^{c}}^{R}}{2}, \quad q_{Q_{\alpha}}^{R}=\frac{q_{D_{\alpha}^{c}}^{R}}{2}, \\
& q_{U_{\alpha}^{c}}^{R}=2-2 q_{D_{\alpha}^{c}}^{R}, \quad q_{E_{\alpha}^{c}}^{R}=-2+3 q_{D_{\alpha}^{c}}^{R} .
\end{aligned}
$$

The $R$ symmetry only has an $S U(2)_{L}$ mixed anomaly with anomaly coefficient $A_{R 22}=-1$, and an $S U(2)_{L}$ mixedanomaly-free $R$ charge can be constructed [11]:

$$
\bar{R}=R+\frac{2}{3 c_{B L}}\left(c_{B} B+c_{L} L\right) .
$$

Gaugino masses $m_{\tilde{g}}$ break the $R$ symmetry explicitly, and by comparing the associated interaction rate $\Gamma_{\bar{R}} \sim m_{\tilde{g}}^{2} / T$ to the Hubble rate $H$, we obtain [10]

$$
T_{\bar{R}} \sim 8 \times 10^{7}\left(\frac{m_{\tilde{g}}}{1 \mathrm{TeV}}\right)^{2 / 3} .
$$

Above this temperature, $U(1)_{\bar{R}}$ is preserved by all the MSSM interactions.

In any extension to the MSSM, one has the freedom to choose $c_{B}$ and $c_{L}$ such that $\bar{P}$ and/or $\bar{R}$ are conserved by the new interactions. ${ }^{8}$ Let us consider a simple model of baryogenesis which breaks $\bar{P}$ and/or $\bar{R}$ without explicitly breaking $B$ and $L$. We introduce a new chiral superfield $S$ uncharged under the SM gauge symmetry with the superpotential $\lambda S H_{u} H_{d}+\frac{1}{2} M S S$. Taking $q_{S}^{\bar{P}}=0$ and $q_{S}^{\bar{R}}=1$, both $\bar{P}$ and $\bar{R}$ are broken by nonzero $\lambda$, by $\Delta \bar{P}=\frac{3}{2}$ and $\Delta \bar{R}=3-2=1$, respectively, while respecting all the $U(1)_{x}$ symmetries in Eq. (8). In this case, there is still an exactly conserved $R$ charge:

$$
R_{c}=\bar{R}-\frac{2}{3} \bar{P} .
$$

Nonzero charges can develop in both $\bar{R}$ and $\bar{P}$ (related by $R_{c}$ ) through $C P$-violating decays $S \rightarrow H_{u} H_{d}$.

Let us consider $(B-L)$ violation from the dimension-7 operator $D_{\alpha}^{c} U_{\beta}^{c} D_{\gamma}^{c} \ell_{\delta} H_{u}$ which can induce nucleon decay

\footnotetext{
${ }^{8} \mathrm{R}$-parity-violating terms $U^{c} D^{c} D^{c}, Q D^{c} L$, and $L L E^{c}$ conserve $P$ and $R$, while one can choose either $c_{B}=0$ or $c_{L}=0$ such that some of them conserve $\bar{P}$ and $\bar{R}$.
}

such as $n \rightarrow e^{-} \pi^{+}$. In order to sufficiently suppress this process, the effective scale of the operator should be of the order of $\gtrsim 10^{11} \mathrm{GeV}$ [4]. With large couplings, the processes mediated by the operator can be in equilibrium at temperature $10^{10} \mathrm{GeV} \lesssim T \lesssim 10^{11} \mathrm{GeV}$. We will take $c_{B}=-2 c_{L}$, such that the operator ${ }^{9}$ conserves $\bar{R}$. This operator violates $U(1)_{\bar{P}}$, and hence $R_{c}$ is no longer conserved. Assuming the operator involves all generations of quarks, all effective symmetries related to quarks are violated. With the remaining conserved charges $(e, \mu, Y)$ being zero, after all $S$ particles have decayed above $T \sim 10^{10} \mathrm{GeV}$, we have

$$
Y_{\Delta(B-L)}=\frac{21}{187} Y_{\Delta \bar{R}}
$$

Below $T_{B-L},(B-L)$ is conserved, and the final baryon asymmetry will be given by Eq. (9) with $Y_{\Delta Y}=0$ and $c_{B(B-L)}=\frac{30}{97}$, assuming at $T_{B-}$ that the thermal bath has the same relativistic degrees of freedom as in the SM.

\section{CONCLUSIONS}

We have categorized all the effective $U(1)$ symmetries and their associate Noether charges in both the SM and the MSSM: 16 in the former, and 18 in the latter. We have demonstrated that, together with fast $(B-L)$-violating interactions, which are ubiquitous in the early Universe, and considering the effective symmetries in the SM or the MSSM above $T_{e} \sim 30 \mathrm{TeV}$, asymmetries can be generated in any of the associated charges through processes that can, but do not have to, violate $B$ and/or $L$.

Since this new avenue of baryogenesis calls for some inequilbrium $(B-L)$-violating interactions in the early Universe, it generally implies an enhanced $\Delta(B-L)=2$ rate for experiments to observe: neutrinoless double beta decay $(\Delta L=2)$, nucleon decay $(\Delta B=-\Delta L=1)$, and neutron-antineutron oscillation $(\Delta B=2)$. A discovery of any of these phenomena will pinpoint the scale where $(B-L)$-violating interactions could be in thermal equilibrium and allow the identification of effective charges to realize baryogenesis above that scale. In the early Universe, $(B-L)$ violation could be the second crucial piece to the puzzle of cosmic baryon asymmetry after the SM baryonnumber violation.

Future direction in this exploration includes identifying new effective symmetries and explicit sources of $(B-L)$ violation that come out from more fundamental theories like grand unified theories. In studying these more specific models to realize the new baryogenesis proposed here, one will be able to have more definite predictions in the rate of $(B-L)$-violating processes.

\footnotetext{
${ }^{9}$ The Weinberg operator conserves $\bar{P}$ and $\bar{R}$, with the choice $c_{B}=-5 c_{L} / 3$.
} 


\section{ACKNOWLEDGMENTS}

C. S.F. acknowledges the support by FAPESP Grant No. 2019/11197-6 and CNPq Grant No. 301271/2019-4. He would like to thank Shaikh Saad for the comments on the manuscript.
Note added.-A similar idea recently appeared in Ref. [44], which considers the dynamics of type-I seesaw as the source of $(B-L)$ violation. Our results, where there are overlaps, are consistent with each other.
[1] G. 't Hooft, Symmetry Breaking Through Bell-Jackiw Anomalies, Phys. Rev. Lett. 37, 8 (1976).

[2] V. A. Kuzmin, V. A. Rubakov, and M. E. Shaposhnikov, On the anomalous electroweak baryon number nonconservation in the early universe, Phys. Lett. 155B, 36 (1985).

[3] P. A. Zyla et al. (Particle Data Group), Review of particle physics, Prog. Theor. Exp. Phys. 2020, 083C01 (2020).

[4] S. Weinberg, Varieties of baryon and lepton nonconservation, Phys. Rev. D 22, 1694 (1980).

[5] B. A. Campbell, S. Davidson, J. R. Ellis, and K. A. Olive, On the baryon, lepton flavor and right-handed electron asymmetries of the Universe, Phys. Lett. B 297, 118 (1992).

[6] J. M. Cline, K. Kainulainen, and K. A. Olive, On the Erasure and Regeneration of the Primordial Baryon Asymmetry by Sphalerons, Phys. Rev. Lett. 71, 2372 (1993).

[7] J. M. Cline, K. Kainulainen, and K. A. Olive, Protecting the primordial baryon asymmetry from erasure by sphalerons, Phys. Rev. D 49, 6394 (1994).

[8] A. Antaramian, L. J. Hall, and A. Rasin, Hypercharge and the cosmological baryon asymmetry, Phys. Rev. D 49, 3881 (1994).

[9] D. A. Sierra, C. S. Fong, E. Nardi, and E. Peinado, Cloistered baryogenesis, J. Cosmol. Astropart. Phys. 02 (2014) 013.

[10] L. E. Ibanez and F. Quevedo, Supersymmetry protects the primordial baryon asymmetry, Phys. Lett. B 283, 261 (1992).

[11] C. S. Fong, Baryogenesis from symmetry principle, Phys. Lett. B 752, 247 (2016).

[12] M. D’Onofrio, K. Rummukainen, and A. Tranberg, Sphaleron Rate in the Minimal Standard Model, Phys. Rev. Lett. 113, 141602 (2014).

[13] See Supplemental Material at http://link.aps.org/ supplemental/10.1103/PhysRevD.103.L051705 for the estimation of the temperatures when the effective symmetries pertaining to quark sector are broken. In addition, we also provide a table of coefficients $c_{(B-L) x}$ in Eq. (10) which relate the $B-L$ charge to the SM effective charges when $B-L$-violating interactions are in thermal equilibrium.

[14] B. Garbrecht and P. Schwaller, Spectator effects during leptogenesis in the strong washout regime, J. Cosmol. Astropart. Phys. 10 (2014) 012.

[15] D. E. Morrissey and M. J. Ramsey-Musolf, Electroweak baryogenesis, New J. Phys. 14, 125003 (2012).

[16] A. D. Sakharov, Violation of $C P$ Invariance, C asymmetry, and baryon asymmetry of the Universe, Sov. Phys. Usp. 34, 392 (1991).
[17] M. B. Gavela, P. Hernandez, J. Orloff, and O. Pene, Standard model $C P$ violation and baryon asymmetry, Mod. Phys. Lett. A 09, 795 (1994).

[18] P. Huet and E. Sather, Electroweak baryogenesis and standard model CP violation, Phys. Rev. D 51, 379 (1995).

[19] K. Kajantie, M. Laine, K. Rummukainen, and M. E. Shaposhnikov, A Nonperturbative analysis of the finite $T$ phase transition in $S U(2) \times U(1)$ electroweak theory, Nucl. Phys. B493, 413 (1997).

[20] J. A. Harvey and M. S. Turner, Cosmological baryon and lepton number in the presence of electroweak fermion number violation, Phys. Rev. D 42, 3344 (1990).

[21] M. Fukugita and T. Yanagida, Baryogenesis without grand unification, Phys. Lett. B 174, 45 (1986).

[22] D. Aristizabal Sierra, M. Losada, and E. Nardi, Variations on leptogenesis, Phys. Lett. B 659, 328 (2008).

[23] D. Aristizabal Sierra, L. A. Munoz, and E. Nardi, Purely flavored leptogenesis, Phys. Rev. D 80, 016007 (2009).

[24] K. S. Babu and R. N. Mohapatra, $B-L$ Violating Proton Decay Modes and New Baryogenesis Scenario in $S O(10)$, Phys. Rev. Lett. 109, 091803 (2012).

[25] K. Dick, M. Lindner, M. Ratz, and D. Wright, Leptogenesis with Dirac neutrinos, Phys. Rev. Lett. 84, 4039 (2000).

[26] H. Murayama and A. Pierce, Realistic Dirac leptogenesis, Phys. Rev. Lett. 89, 271601 (2002).

[27] P.-H. Gu, From Dirac neutrino masses to baryonic and dark matter asymmetries, Nucl. Phys. B872, 38 (2013).

[28] K. Earl, C. S. Fong, T. Gregoire, and A. Tonero, Mirror Dirac leptogenesis, J. Cosmol. Astropart. Phys. 03 (2020) 036.

[29] A. E. Nelson and S. M. Barr, Upper bound on baryogenesis scale from neutrino masses, Phys. Lett. B 246, 141 (1990).

[30] F. F. Deppisch, J. Harz, and M. Hirsch, Falsifying HighScale Leptogenesis at the LHC, Phys. Rev. Lett. 112, 221601 (2014).

[31] F. F. Deppisch, J. Harz, M. Hirsch, W.-C. Huang, and H. Päs, Falsifying high-scale baryogenesis with neutrinoless double beta decay and lepton flavor violation, Phys. Rev. D 92, 036005 (2015).

[32] F. F. Deppisch, L. Graf, J. Harz, and W.-C. Huang, Neutrinoless double beta decay and the baryon asymmetry of the Universe, Phys. Rev. D 98, 055029 (2018).

[33] M. Fukugita and T. Yanagida, Sphaleron induced baryon number nonconservation and a constraint on Majorana neutrino masses, Phys. Rev. D 42, 1285 (1990).

[34] M. Fukugita and T. Yanagida, Resurrection of Grand Unified Theory Baryogenesis, Phys. Rev. Lett. 89, 131602 (2002). 
[35] S. Weinberg, Baryon and Lepton Nonconserving Processes, Phys. Rev. Lett. 43, 1566 (1979).

[36] H.-L. Li, Z. Ren, J. Shu, M.-L. Xiao, J.-H. Yu, and Y.-H. Zheng, Complete set of dimension- 8 operators in the standard model effective field theory, arXiv:2005.00008.

[37] L. Lehman, Extending the standard model effective field theory with the complete set of dimension-7 operators, Phys. Rev. D 90, 125023 (2014).

[38] Y. Liao and X.-D. Ma, Renormalization group evolution of dimension-seven baryon- and lepton-number-violating operators, J. High Energy Phys. 11 (2016) 043.

[39] H.-L. Li, Z. Ren, M.-L. Xiao, J.-H. Yu, and Y.-H. Zheng, Complete set of dimension-9 operators in the standard model effective field theory, arXiv:2007.07899.
[40] Y. Liao and X.-D. Ma, An explicit construction of the dimension-9 operator basis in the standard model effective field theory, J. High Energy Phys. 11 (2020) 152.

[41] K.S. Babu and C. N. Leung, Classification of effective neutrino mass operators, Nucl. Phys. B619, 667 (2001).

[42] A. de Gouvea and J. Jenkins, A survey of lepton number violation via effective operators, Phys. Rev. D 77, 013008 (2008).

[43] D. G. Phillips II et al., Neutron-antineutron oscillations: Theoretical status and experimental prospects, Phys. Rep. 612, 1 (2016).

[44] V. Domcke, K. Kamada, K. Mukaida, K. Schmitz, and M. Yamada, Wash-In Leptogenesis, arXiv:2011.09347. 